\title{
PCPT, MTOPS and the use of 5ARIs: a Canadian consensus regarding implications for clinical practice
}

\author{
Laurence Klotz, MD, FRCSC; ${ }^{*}$ Fred Saad, MD, FRCSC; ${ }^{\dagger}$ \\ and the PCPT-MTOPS Consensus Panel
}

See related article on page 22

\begin{abstract}
Objectives: Two large, recently published, definitive trials evaluated the benefits of 5-alpha reductase inhibitors (5ARIs). The Prostate Cancer Prevention Trial (PCPT) tested the effect of finasteride for prostate cancer prevention and the Medical Therapy of Prostatic Symptoms (MTOPS) tested its effect in benign prostatic hyperplasia (BPH). Both trials were strongly positive. However, the role of 5ARIs in the clinical management of patients remains controversial. The consensus conference, which forms the basis for this report, attempted to develop an expert opinion, based on these studies, as to the optimal use of 5ARIs in patient management.
\end{abstract}

Methods: The Canadian Consensus Meeting, organized by the Canadian Urology Research Consortium and the Canadian Urologic Oncology Group, held in Toronto on May 7, 2006, focused on the new data from the PCPT and the MTOPS study. Internationally recognized experts and clinicians discussed the implications of these data on clinical practice and issued a recommendation on the optimal management of patients with $\mathrm{BPH}$.

Results: The Consensus meeting agreed on the following recommendations:

1. The overall results from the PCPT and MTOPS studies are of importance to the urologic, as well as to the greater medical, community.

2. Prostate management guidelines should be updated to include the results from both the MTOPS and the PCPT studies.

3 . In the PCPT, the incidence of high-grade cancer was higher in the finasteridetreated group (6.4\%), compared with the placebo group (5.1\%). Subsequent analyses strongly suggest that this increased prevalence was owing to a detection bias caused by the reduction in prostate volume in patients taking finasteride, compared with patients taking placebo. This resulted in an improved detection at biopsy of high-grade cancer in the finasteride group.

4. In men who have large prostates and lower urinary tract symptoms (LUTS), 5 ARIs ${ }^{\S}$ should be considered, both for the treatment of BPH and for prostate cancer risk reduction.

5. For men who are concerned about prostate cancer, it is appropriate to discuss chemoprevention with finasteride.

6. Urologists are encouraged to disseminate these recommendations among other healthcare professionals.

CUAJ 2007;1(1):17-21

SProstate cancer risk reduction has been demonstrated in PCPT with finasteride; however, a class effect with respect to dutasteride has not yet been established.
$\mathrm{T}$ wo large, recently published, definitive trials evaluated the benefits of 5 -alpha reductase inhibitors (5ARIs). The Prostate Cancer Prevention Trial (PCPT) tested the effect of finasteride for prostate cancer prevention ${ }^{1}$ and the Medical Therapy of Prostatic Symptoms (MTOPS) study tested its effect in benign prostatic hyperplasia (BPH). ${ }^{2}$ Both trials were strongly positive. However, the role of 5ARIs in the clinical management of patients remains controversial and practice patterns vary widely. The consensus conference, which forms the basis for this report, attempted to develop an expert opinion, based on these studies, as to the optimal use of 5 ARIs in patient management.

There are a number of reasons why practice patterns vary widely with respect to 5ARI use:

- The PCPT showed a 1.3\% increase in highgrade cancer in the finasteride-treated group. The concern about an adverse biological effect of finasteride on prostate cancer has inhibited the widespread use of the drug for prostate cancer prevention.

- The MTOPS study evaluated the benefit of finasteride, alone and in combination with doxazosin, in the management of $\mathrm{BPH}$. The PCPT evaluated the benefit of finasteride in prostate cancer prevention. Patients, however, often have both $\mathrm{BPH}$-lower urinary tract symptoms (LUTS) and prostate cancer concerns. There has been no consensus as to how the respective benefits of $\mathrm{BPH}$ treatment and prostate cancer prevention should be integrated in individual patients.

- Urologists have historically been geared toward diagnosis and therapeutic intervention. The concept of disease prevention has not been a robust part of our tradition. 
- Some of the data, particularly regarding prostate-specific antigen (PSA) as a surrogate for $\mathrm{BPH}$ and prostate cancer risk, and the interpretation of PSA in men on 5ARIs, has been complex. This might have resulted in some reluctance to initiate therapy.

- Many primary care physicians are unfamiliar with the data, concerned about missing prostate cancer, suspicious of PSA as a screening test and reluctant to take on a new domain in patient care. Accordingly, the authors believe that a consensus meeting involving experts in the field and including methodologists with a clinical epidemiology background was warranted.

\section{Methods}

The Canadian Consensus Meeting, organized by the Canadian Urology Research Consortium (CURC) and the Canadian Urologic Oncology Group (CUOG), was held in Toronto on May 7 2006. It focused on the new data from the PCPT and the MTOPS study. Recognized experts and clinicians discussed the implications of these data on clinical practice and issued a recommendation on the optimal management of patients with $\mathrm{BPH}$. Invited presenters reviewed the literature and presented expert opinions. In contrast to formal treatment guidelines, these recommendations are

- focused on specific issues and relevant questions of daily clinical practice;

- meant to be promptly available to the scientific community through a simple and fast development process; and

- representative of a consensus of expert opinions based on published evidence rather than a formal, explicit review of this evidence.

This consensus meeting was held in camera, with representation from prostate cancer and BPH experts in urology academic practice and community practice, as well as clinical epidemiologists and primary care. There were no pharmaceutical company representatives involved in the planning or implementation of the meeting program, nor were they present during the consensus meeting itself (see Acknowledgements).

The key references used by the panel are listed in the References. ${ }^{1-13}$

\section{Results}

\section{PCPT}

The landmark PCPT was the largest completed and published intervention study conducted in the urologic community. It reported, for the first time, that medical intervention reduced the incidence of diagnosed prostate cancer to a clinically meaningful extent. An overview of the findings from the PCPT and the MTOPS study, as well as the following, formed the basis for discussion and consensus development:

- Prostate cancer is one of the leading causes of cancer death in Canada.

- The MTOPS study compared doxazosin, finasteride and the combination with placebo in a large 4-arm study, which showed that in men with prostatic enlargement, the combination of the 2 drugs significantly reduced the risk of urinary retention, surgery for $\mathrm{BPH}$ and significant progression of symptoms, compared with either drug alone.

- The PCPT is the largest intervention study ever reported in the urologic community. This study was initiated and funded by the National Cancer Institute of the USA National Institutes of Health. The PCPT reports, for the first time, that medical intervention reduces the incidence of diagnosed prostate cancer to a clinically meaningful extent (level 1B evidence).

- The results showed that finasteride reduced the risk of detecting prostate cancer by $25 \%$, compared with placebo $(24.4 \%$ placebo v. $18.4 \%$ finasteride). The risk was reduced in all subgroups, analyzed by age, ethnicity, 
presence of family history and baseline PSA level.

- The finasteride-treated group was found to have a higher proportion of highgrade cancers $(6.4 \%)$, compared with the placebo group $(5.1 \%)(p<0.001)$. Subsequent analyses have concluded, with a high degree of confidence, that this increased prevalence was owing wholly or in great part to a detection bias. This was caused by the following effects:

1. The reduction in prostate volume in patients taking finasteride, compared with patients taking placebo. The median prostate volume was $24 \%$ lower in patients in the finasteride arm, compared with those in the placebo arm (25.5 mL v. $33.6 \mathrm{~mL})$. This resulted in improved detection accuracy at biopsy of high-grade cancer in the finasteride group.

2. The improved performance of PSA in the finasteride arm, relative to placebo. ROC (receiver operating characteristic) analyses have been conducted on the biomarker PSA. ${ }^{3}$ The area under the curve values for placebo and finasteride-treated patients indicated that PSA performed better with respect to overall detection and detection of highgrade cancers in finasteride-treated patients, compared with those receiving placebo. This further biased the study toward the detection of high-grade cancer in the finasteride group.

The increase in high-grade tumours on biopsy was seen within 1 year of finasteride exposure and did not increase over time. Analysis of the radical prostatectomy specimens from the PCPT trial patients has shown no difference in grade, pathologic stage, perineural invasion or any other parameter. An independent report from a large biopsy radical prostatectomy database confirmed that the upgrading rate from biopsy to radical prostatectomy specimen is higher for large volume glands. ${ }^{4}$ Taken together, this provides convincing evidence for the artifactual nature of the upgrading seen in the PCPT.

The impact of the reduction in incidence of prostate cancer using finasteride has been modelled based on the PCPT. ${ }^{5}$ A $24.8 \%$ reduction in the incidence of prostate cancer for 5 years among men from the United States, aged 55 years and older would lead to an estimated saving of 316760 person-years due to finasteride. Allowing for an absolute increase of $6.9 \%$ in the proportion of men with high-grade tumours (corresponding to the difference between the rates on the placebo and finasteride arms of the PCPT), the number of person-years saved would be reduced to 262567 . Each absolute increase of $5 \%$ in the proportion of patients with high-grade tumours would reduce the number of person-years saved by 39000 . This reinforces the view that the widespread use of finasteride for prevention would have a major impact on population mortality from prostate cancer. In the unlikely event that the high Gleason scores represented an adverse biological effect of finasteride, the detrimental effects of an increased rate of high-grade prostate cancer would still be substantially outweighed by the reduction in overall incidence.

A theoretical limitation of the PCPT data is that prostate cancer mortality was not an end point. This is a characteristic of primary prevention trials in general. There was a consensus that a $25 \%$ reduction in the rate of diagnosis, which would result in a significant decrease in the need for treatment and its attendant morbidities and costs, was an important clinical end point. Patients in the trial who were diagnosed with prostate cancer have been treated and will likely be cured. This treatment effect means that it is unlikely that longer-term follow-up will show a difference in prostate cancer mortality.

\section{MTOPS}

The MTOPS study demonstrated that the combination of finasteride and doxazosin 
provided greater benefits than either agent alone. Recent analyses of the MTOPS study indicate that patients with prostates greater than $25 \mathrm{~mL}$ may benefit from combination therapy. Similarly, those with a baseline prostate volume greater than $30 \mathrm{~mL}$ are at significantly greater risk of progression.

The PCPT also demonstrated the positive effects of finasteride with respect to genitourinary symptoms, with a significantly $(p<0.01)$ lower incidence of BPH, prostatitis, urinary tract infection for urgency or frequency, urinary retention and transurethral resection of the prostate procedures, compared with patients receiving placebo. These, along with the additional benefit of the reduced risk of developing prostate cancer, have to be balanced against an increase in 5ARIrelated side effects, including reduced ejaculate volume, decreased libido and erectile dysfunction.

The use of finasteride to treat men with $\mathrm{BPH}$ enhances the use of PSA in detecting prostate cancer. Finasteride decreases PSA by about $50 \%$ during the first 12 months of use. As noted above, PSA performs better in detecting prostate cancer in general and high-grade cancer, specifically in men treated with finasteride.

\section{Consensus Statements}

On the basis of this evidence, we agreed upon the following statements:

1. Implications for LUTS-BPH management:

a. The 5ARIs finasteride and dutasteride are appropriate and effective treatments for patients with LUTS associated with demonstrable prostatic enlargement (greater than $30 \mathrm{~mL}$ ). In patients without prostate cancer, a PSA value greater than 1.5 may be a useful marker for prostatic enlargement.

b. If one assumes a class effect, all combinations of an a1-adrenoceptor antagonist and a 5ARI would be equally effective. c. The most robust data supporting the safety and efficacy of the combination used doxazosin and finasteride.

d. Based on current knowledge, a PSA conversion factor of about 2 for patients taking $5 \mathrm{ARI}$ is required to interpret the test according to accepted cut-off levels. The PSA requires about 6 months to achieve a nadir after 5ARIs are introduced. Further analysis of the PCPT data suggests that finasteride therapy improves the specificity and sensitivity of the PSA test, and enhances detection of high-grade disease.

e. It is recommended that prostate management guidelines are updated to include the results from these and further studies.

2. Implications for prostate cancer chemoprevention:

a. The PCPT showed that finasteride significantly reduces the prevalence of histologically proven prostate cancer.

b. In men who have large prostates and LUTS, $5 \mathrm{ARIs}^{\S}$ should be considered, both for treatment of $\mathrm{BPH}$ and to reduce prostate cancer risk.

c. For men who are concerned about prostate cancer, it is appropriate to discuss chemoprevention with finasteride.

d. In doing the above, it is important to highlight both the benefits and the risks associated with long-term treatment.

3. Implications for patient information:

a. Physicians should be aware of the findings from the PCPT and the MTOPS study.

b. Patients treated with 5ARIs for chemoprevention should be monitored for prostate cancer.

4. Implications for the education of the medical community:

\$Prostate cancer risk reduction has been demonstrated in PCPT with finasteride; however, a class effect with respect to dutasteride has not yet been established. 
a. Urologists are encouraged to disseminate these recommendations among other healthcare professionals, including general practitioners who often play a significant role in the management of BPH-LUTS.

b. Physicians should appropriately counsel their patients about the potential benefits and side effects of treatment with 5ARIs.

c. Management guidelines on prostate disease should be updated to reflect the findings from the PCPT and the MTOPS study.

\section{Conclusions}

The MTOPS and the PCPT, 2 of the most important and definitive studies published in the field in the last 5 years, have major implications for the management of men with LUTS-BPH who do, as well as those who do not, have concerns or risk factors for prostate cancer. We achieved a Canadian consensus on the role of 5ARIs in the management of these men. They should be presented with the benefits and the risks of taking a $5 \mathrm{ARI}$ and should be assisted in integrating their urinary and sexual symptoms, prostate cancer risk factors and level of concern into their decisionmaking process.

From the ${ }^{*}$ Division of Urology, Sunnybrook Health Sciences Centre, Toronto, Ont. and the 'Department of Urologic Oncology, Centre Hospitalier de l'université de Montréal, Montréal, Que.

Acknowledgements: This consensus conference was funded by an unrestricted educational grant from Merck Canada to the Canadian Urology Research Consortium (CURC) and the Canadian Urologic Oncology Group (CUOG). The conference was planned, organized, and implemented completely independently by CURC and CUOG.

Participants in the CURC-CUOG PCPT-MTOPS Consensus Conference, May 7 2006, included Dr. Armen Aprikian, Dr. Scott Bagnall, Dr. Jack Barkin, Dr. Michael Chetner, Dr. Joseph Chin, Dr. Mostafa Elhilali, Dr. Larry Goldenberg, Dr. Steven Grover, Dr. Michael Jewett, Dr. Anil Kapoor, Dr. Pierre Karakiewicz, Dr. Laurence Klotz, Dr. Ross MacMahon, Dr. Chris Morash, Dr. Joel Nelson, Dr. Curtis Nickel, Dr. Paul Perrotte, Dr. Ricardo Rendon, Dr. Fred Saad, Dr. lan Thompson, Dr. John Trachtenberg, Dr. Claude Trudel, Dr. Ross Upshur and Dr. Luc Valiquette.

This article has been peer reviewed.

\section{Competing interests: None declared.}

\section{References}

1. Thompson IM, Goodman PJ, Tangen CM, et al. The influence of finasteride on the development of prostate cancer. N Engl J Med 2003;349: 215-24.

2. McConnell JD, Roehrborn CG, Bautista OM, et al. Medical Therapy of Prostatic Symptoms (MTOPS) Research Group. The long-term effect of doxazosin, finasteride, and combination therapy on the clinical progression of benign prostatic hyperplasia. N Engl J Med 2003;349: 2387-98.

3. Thompson IM, Chi C, Ankerst DP, et al. Effect of finasteride on the sensitivity of PSA for detecting prostate cancer. J Natl Cancer Inst 2006; 98:1128-33.

4. Kulkarni GS, Al-Azab R, Lockwood G, et al. Evidence for a biopsy derived grade artifact among larger prostate glands. J Urol 2006; 175:505-9.

5. Unger JM, Thompson IM Jr, LeBlanc $M$, et al. Estimated impact of the Prostate Cancer Prevention Trial on population mortality. Cancer 2005;103:1375-80.

6. Thompson IM, Ankerst DP, Chi C, et al. Assessing prostate cancer risk: results from the Prostate Cancer Prevention Trial. J Natt Cancer Inst 2006;98:529-34.

7. Thompson IM, Pauler DK, Goodman PJ, et al. Prevalence of prostate cancer among men with a prostate-specific antigen level $<$ or $=4.0$ ng per milliliter. $N$ Engl J Med 2004;350:2239-46.

8. Grover S, Lowensteyn I, Hajek D, et al. Do the benefits of finasteride outweigh the risks in the prostate cancer prevention trial? I Urol 2006; 175:934-8.

9. Goodman PJ, Thompson IM Jr, Tangen $C M$, et al. The prostate cancer prevention trial: design, biases and interpretation of study results. J Urol 2006; 175:2234-42

10. Kaplan SA, McConnell JD, Roehrborn CG, et al. Medical Therapy of Prostatic Symptoms (MTOPS) Research Group. Combination therapy with doxazosin and finasteride for benign prostatic hyperplasia in patients with lower urinary tract symptoms and a baseline total prostate volume of $25 \mathrm{ml}$ or greater. J Urol 2006;175:217-20.

11. McDonald H, Hux M, Brisson M, et al. An economic evaluation of doxazosin, finasteride and combination therapy in the treatment of benign prostatic hyperplasia. Can J Urol 2004;11:2327-40.

12. Turner K, McNeill SA. MTOPS: conclusions about invasive therapy for BPH should be interpreted with caution. BJU Int 2004;94:1143.

13. Madersbacher S, Alivizatos G, Nordling J, et al. EAU 2004 guidelines on assessment, therapy and follow-up of men with lower urinary tract symptoms suggestive of benign prostatic obstruction (BPH guidelines). Eur Urol 2004;46:547-54.

Correspondence: Dr. Laurence Klotz, Division of Urology, Sunnybrook Health Sciences Centre, 2075 Bayview Ave., \#MG408, Toronto 0N M4N 3M5; laurence.klotz@sunnybrook.ca 